\title{
Nanometer-scale studies of vertical organization and evolution of stacked self-assembled InAs/GaAs quantum dots
}

\author{
B. Lita and R. S. Goldman ${ }^{\text {a) }}$ \\ Department of Materials Science and Engineering, University of Michigan, Ann Arbor, \\ Michigan 48109-2136 \\ J. D. Phillips and P. K. Bhattacharya \\ Solid State Electronics Laboratory, University of Michigan, Ann Arbor, Michigan 48109-2122
}

(Received 17 December 1998; accepted for publication 11 March 1999)

\begin{abstract}
We have investigated the vertical organization and evolution of 1-, 5-, 10-, and 20-layer stacks of molecular beam epitaxially grown self-assembled InAs/GaAs quantum dots using high resolution and large-scale cross-sectional scanning tunneling microscopy. We report results regarding the evolution of the dot sizes and shapes, and the assembly of vertically organized columns of stacked dots. As the number of dot layers within a stack is increased, the average spacing between vertically organized columns decreases, and the corresponding dots become more uniform in size. The data also suggest that the coalescence of neighboring stacks of dots has not occurred and therefore coalescence is not the mechanism leading to the observed uniform distribution of dot sizes and column spacings. (C) 1999 American Institute of Physics. [S0003-6951(99)02619-4]
\end{abstract}

The formation of quantum dots during epitaxial growth $^{1-3}$ has gained considerable interest in recent years due to the ease with which low-dimensional structures can be produced by self-organized growth in comparison with postgrowth lithographic patterning. Dense arrays of uniformly sized dots are required for both electronic and photonic applications. ${ }^{4}$ Meanwhile, variations in the size and shape of such dots significantly impact their electronic structure and luminescence properties. ${ }^{5}$ Hence, the nanometer-scale details of the uniformity of dot organization, as well as the evolution of dot shapes and sizes in capped self-assembled quantum dots are critical for the development of novel photonic and electronic applications. Therefore, we have investigated the vertical organization and evolution of 1-, 5-, 10-, and 20layer stacks of molecular beam epitaxially (MBE) grown capped self-assembled InAs/GaAs quantum dots using ultrahigh vacuum (UHV) cross-sectional scanning tunneling microscopy (XSTM). In this letter, we report results regarding the mechanisms and the lateral uniformity of selforganization in the InAs/GaAs system. High resolution and large-scale images show that the stacked InAs/GaAs quantum dots have self-organized into columns, with the vertical column axis primarily along the [001] growth direction, similar to earlier reports. ${ }^{5-13}$ However, in some cases, the vertical column axis is rotated towards the [110] or [ $\overline{1} \overline{1} 0]$ direction. As the number of dot layers in the stack is increased, the linear density of vertically organized columns increases, and the average spacing between vertically organized columns decreases. Surprisingly, the spacing between the columns is constant throughout the thickness of a particular stack of dots. In the 1-layer stacks, the dots have an oval shape. In both the 5- and 10-layer stacks, the dot shapes become progressively less symmetric about [110], and the dot sizes increase monotonically towards the top. In the 10layer stacks, the top few dot layers are more uniformly sized and often develop distinct facets. In addition, neighboring

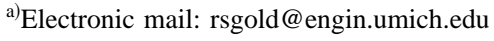

vertically organized columns occasionally appear close together, but unlike previous reports, ${ }^{12,13}$ these columns have not coalesced.

The samples were grown by solid source MBE, using an $\mathrm{As}_{4}$ source. The 1-, 5-, 10-, and 20-period dot stacks consisting of 2.6 ML InAs and $5 \mathrm{~nm} \mathrm{GaAs}$ were grown on Si-doped $\left(n \sim 3 \times 10^{18} \mathrm{~cm}^{-3}\right)$ GaAs $(001)$ substrates. ${ }^{14}$ In order to prevent the interaction of strain fields from different dot stacks, each stack was separated by a $140 \mathrm{~nm}$ multilayer consisting of a $40 \mathrm{~nm}$ total thickness AlAs/GaAs short-period superlattice sandwiched between two $50 \mathrm{~nm}$ GaAs layers, which we refer to as an isolation layer. The dots and isolation layers were grown at 510 and $620^{\circ} \mathrm{C}$, respectively. In preparation for XSTM, the samples were thinned to $\sim 150 \mu \mathrm{m}$ using conventional mechanical polishing from the backside. For XSTM, the samples were cleaved to expose a (110) surface, in a UHV chamber with base pressure $<5 \times 10^{-11}$ Torr. STM was performed with both electrochemically etched polycrystalline $\mathrm{W}$ and commercially available $\mathrm{Pt}-\mathrm{Ir}$ tips. The tips were cleaned in situ by electron bombardment. All images were obtained with a constant tunnel current of $0.2 \mathrm{nA}$ and sample bias voltages as described below.

Figures 1(a) and 1(b) show large-scale XSTM topographic images of 1-, 5-, and 10-layer dot stacks, displayed with the growth direction from the bottom to the top. To date, we have not obtained images of the 20-layer dot stacks, presumably because the cleaved surface is not atomically flat in that region. In Fig. 1(b), 1- and 5-layer dot stacks, separated by an isolation layer, are observed in the bottom and top of the image. Within each dot stack, bright ellipses of InAs sandwiched between darker layers corresponding to GaAs are observed. Similarly, in Fig. 1(a), 5- and 10-layer dot stacks, separated by an isolation layer, are observed in the bottom and top of the image. In these dot stacks, bright ellipses of InAs sandwiched in GaAs are also observed. In Figs. 1(a) and 1(b), both the 5- and 10-layer stacks have self-organized into columns, with the vertical axis of the column primarily along the [001] growth direction. Occasion- 

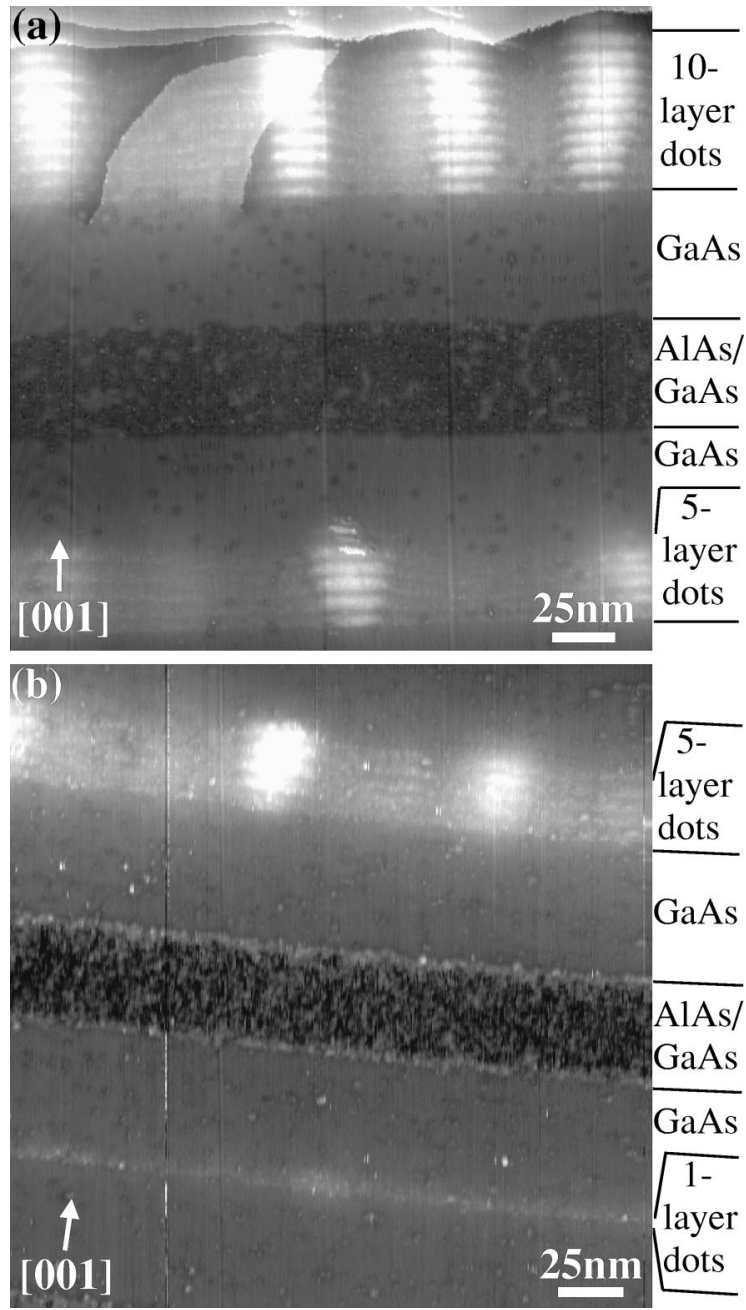

FIG. 1. Large-scale topographic images showing (a) 10- and 5-layer and (b) 5- and 1-layer stacks of InAs/GaAs quantum dots. The images were acquired at sample bias voltages of (a) $-2.5 \mathrm{~V}$ and (b) $-2.2 \mathrm{~V}$. The gray-scale ranges displayed are (a) $13.6 \AA$ and (b) $10.5 \AA$

ally, the vertical column axis of a dot stack is rotated towards the [110] or [ $\overline{1} \overline{1} 0]$ direction. This occurs most often towards the top of a 10-layer dot stack. The vertical column axis rotation occurs in two different ways. In some cases, the normal of an individual dot is rotated $7^{\circ}-11^{\circ}$ away from the [001] direction. In other cases, the normal of the dot remains in the [001] direction, and instead a misalignment in dot stacking has occurred, where the center of the dot appears offset $5-10 \mathrm{~nm}$ laterally (towards the [110] or [ $\overline{1} \overline{1} 0]$ directions) from the center of a dot beneath it.

It is apparent from both Figs. 1(a) and 1(b) that the spacing between vertically organized columns decreases with increasing number of stacked dot layers, similar to theoretical predictions. ${ }^{15}$ In particular, the average spacing between vertical columns decreases by a factor of 1.5 for the 5-layer stacks in comparison with the 1-layer stack. Similarly, the average spacing between vertical columns decreases by a factor of 1.5 for the 10-layer stacks in comparison with the 5-layer stacks. Interestingly, this spacing between the columns is constant throughout the thickness of a particular stack of dots. However, during deposition, a dot does not know a priori whether it will be part of a 1-, 5-, or 10-layer stack. Furthermore, the initial GaAs/dot interface in each
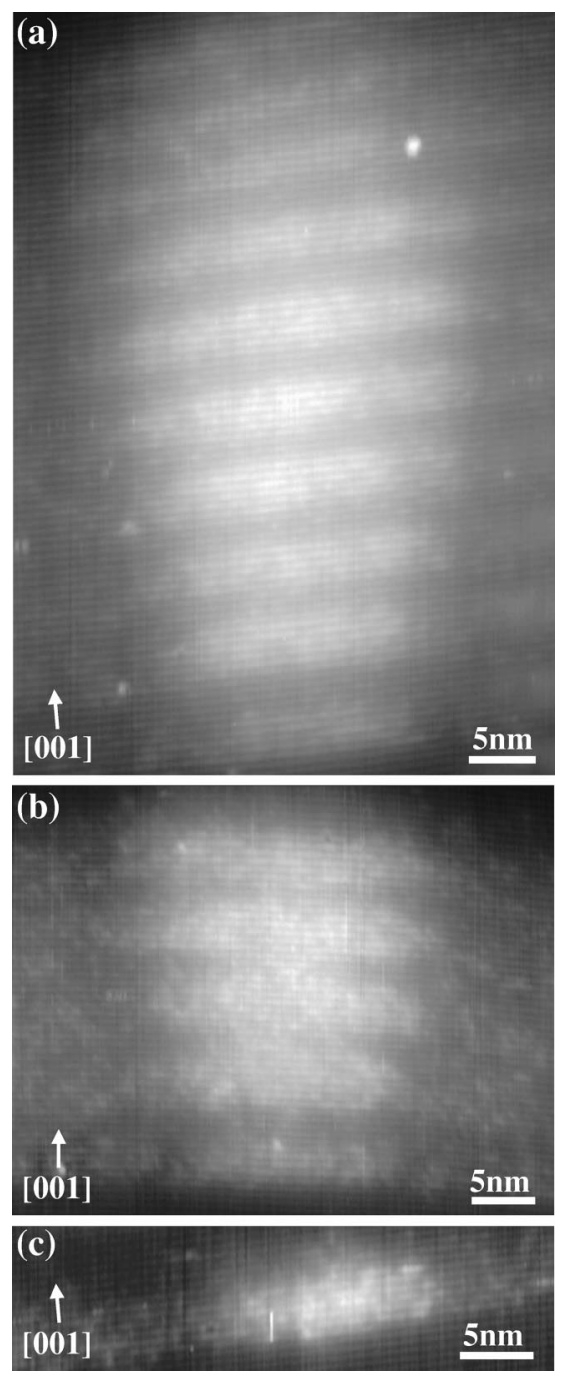

FIG. 2. High resolution topographic images of (a) 10-, (b) 5-, and (c) 1-layer stacks of InAs/GaAs quantum dots, obtained at sample bias voltages of (a) $-2.2 \mathrm{~V}$, (b) $-2.5 \mathrm{~V}$, and (c) $-2.2 \mathrm{~V}$. The gray-scale ranges displayed are (a) $9.7 \AA$, (b) $7.3 \AA$, and (c) $4.0 \AA$.

stack is atomically abrupt. Consequently, the average spacing between the self-organized columns is unlikely to be determined during dot deposition; instead, bulk diffusion processes (occurring primarily during the growth of the isolation layer following each stack ${ }^{16}$ ) are likely to be determining this length-scale. An estimation of bulk In-Ga interdiffusion based upon literature values ${ }^{17}$ results in diffusion lengths significantly smaller than the spacing between the 5and 10-layer dot columns. However, when the effects of strain are considered, ${ }^{18,19}$ the diffusion lengths are similar to the spacing between dot columns, suggesting that this effect is enhanced by strain.

The evolution of the dot sizes and shapes is revealed in Fig. 2, which contains high resolution images of (a) 10-, (b) 5-, and (c) 1-layer stacks of dots. In Figs. 2(a)-2(c), fringes with a spacing of 5.65 and $6.06 \AA$, corresponding to the (001) planes of GaAs and InAs, are observed in the darker and brighter regions of the image, respectively. In Fig. 2(c), the brighter oval shaped region corresponds to a typical 1-layer InAs dot, which is asymmetric about the [110] axis, with a higher radius of curvature in the upward [001] than in the downward [001] direction. Although most of the 1-layer 
dots have a similar shape, symmetric ellipse-shaped 1-layer dots are also occasionally observed. In general, the 1-layer dots have diameters ranging from 16 to $21 \mathrm{~nm}$, and heights ranging from 4.5 to $7 \mathrm{~nm}$, similar to earlier XTEM reports. ${ }^{6,7,14}$

In the 5-layer dot stack, shown in Fig. 2(b), the dots near the bottom of the stack have a symmetric ellipse shape, with diameters similar to the 1-layer dots. The apparent lateral extension (or diameter) of the dots in the 5-layer stack increases monotonically along the [001] growth direction. The maximum dot diameter at the top of the 5-layer stack ranges from 20 to $25 \mathrm{~nm}$. The apparent increase in dot diameter is balanced by depletion of InAs from the wetting layers between the vertical columns of stacked dots. Towards the top of the 5-layer stacks, the dot shapes become progressively less symmetric about the [110] direction, and distinct facets are often observed. In particular, the angles between the (001) plane normal and the normal to the InAs/GaAs interface profile range from $11^{\circ}$ to $32^{\circ}$. Assuming that the faceted surfaces are normal to the cleaved surface, the observed angles correspond to (112) to (117) facets.

In the 10-layer dot stack, shown in Fig. 2(a), the dots near the bottom have a symmetric ellipse shape, with apparent lateral extension similar to the 1- and 5-layer dots. The dot diameter in the 10-layer stack increases monotonically along the [001] growth direction until sometime after the 5th layer, when it reaches a $30-34 \mathrm{~nm}$ saturation. Similar to the 5-layer dot stacks, the increase in dot diameter is balanced by the depletion of InAs from the wetting layers. Also, near the top of the 10-layer stacks, the dots become less symmetric about the [110] direction, and sometimes develop a crescent shape. Near the top of the 10-layer stack, most of the angles between the normal to the (001) plane and that of the InAs/ GaAs interface profile range from $10^{\circ}$ to $15^{\circ}$, corresponding to (115) to (118) facets.

Figure 3 shows an image of two adjacent columns of 10-layer dot stacks, where 5 and 6 dot layers of each stack are displayed. These columns of dot stacks appear close together, yet they have not coalesced. Note that it is likely that these two columns would appear coalesced in a transmission electron microscopy (TEM) image, as has been shown in an earlier report, ${ }^{13}$ due to the averaging through the thickness of the foil inherent in TEM. In many similar large-scale images, we observe a uniform distribution of dot sizes and column spacings at the top of 10-layer stacks of dots, yet we have not observed the coalescence of these columns of dot stacks. Hence, we conclude that the coalescence of dots from neighboring 10-layer stacks is not the mechanism leading to the observed uniform distribution of dot sizes and column spacings. Instead, the uniform distribution of dot sizes and column spacings is likely to be influenced by strain-enhanced bulk interdiffusion processes. In Fig. 3, it is also interesting to note that the dots from adjacent columns are stacked offset from one another along the [001] direction such that an InAs dot of column A is lined up with a GaAs cap of column B. This effect may be due in part to roughening of the surface occurring during the growth of the 10-layer dot stack. Assuming that the average dot diameters in each column are similar, we expect that the center axis of each column is located at a different depth below the cleaved (110) surface.

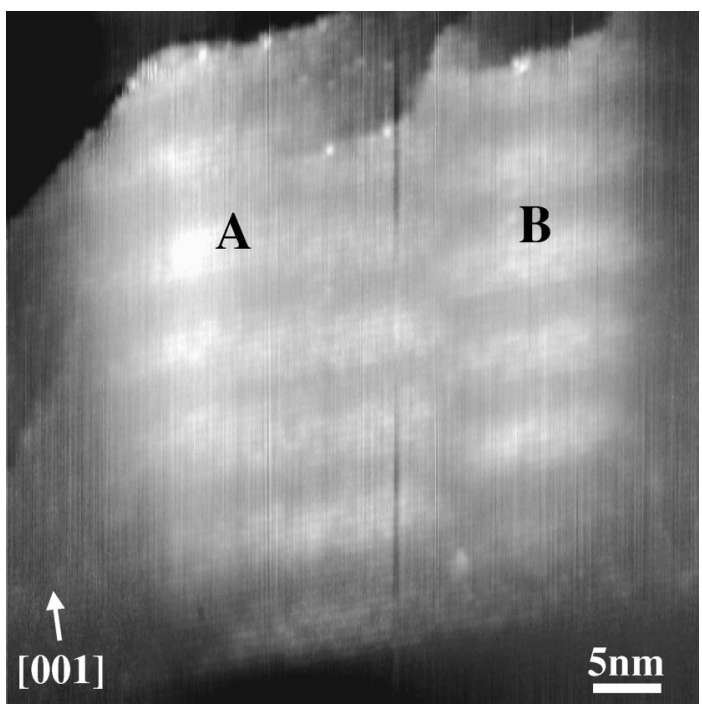

FIG. 3. Topographic image of two adjacent 10-layer dot stacks, showing 5 and 6 dot layers of stacks A and B. The image was acquired at a sample bias voltage of $-2.5 \mathrm{~V}$ and the gray-scale range displayed is $5.5 \AA$.

This work was supported in part by the Office of the Vice President for Research and the Rackham Graduate School at the University of Michigan, the donors of the Petroleum Research Fund, administered by the American Chemical Society, the Dow Corning Foundation, the National Science Foundation (Grant Nos. DMR-9733707 and ECS-9628973), and the Army Research Office (Grant No. DAAH-04-96-1-0001).

${ }^{1}$ L. Goldstein, F. Glas, J. Y. Marzin, M. N. Charasse, and G. Leroux, Appl. Phys. Lett. 47, 1099 (1988).

${ }^{2}$ P. R. Berger, K. Chang, P. Bhattacharya, J. Singh, and K. K. Bajaj, Appl. Phys. Lett. 53, 684 (1988).

${ }^{3}$ D. Leonard, M. Krishnamurthy, C. M. Reaves, S. P. Denbaars, and P. M. Petroff, Appl. Phys. Lett. 63, 3203 (1993).

${ }^{4}$ D. Bimberg, M. Grundmann, and N. N. Ledenstov, Mater. Res. Bull. 23, 31 (1998).

${ }^{5}$ I. Mukhametzhanov, R. Heitz, J. Zeng, P. Chen, and A. Madhukar, Appl. Phys. Lett. 73, 1841 (1998).

${ }^{6}$ Q. Xie, A. Madhukar, P. Chen, and N. P. Kobayashi, Phys. Rev. Lett. 75, 2542 (1995)

${ }^{7}$ S. Rouvimov, Z. Liliental-Weber, W. Swider, J. Washburn, E. R. Weber, A. Sasaki, A. Wakahara, Y. Furkawa, T. Abe, and S. Noda, J. Electron. Mater. 27, 427 (1998).

${ }^{8}$ G. S. Solomon, J. A. Trezza, A. F. Marshall, and J. S. Harris, Jr., Phys. Rev. Lett. 76, 952 (1996)

${ }^{9}$ C. Teichert, M. G. Lagally, L. J. Peticolas, J. C. Bean, and J. Tersoff, Phys. Rev. B 53, 16334 (1996).

${ }^{10}$ G. S. Solomon, S. Komarov, J. S. Harris, Jr., and Y. Yamamoto, J. Cryst. Growth 175/176, 707 (1997).

${ }^{11}$ W. Wu, J. R. Tucker, G. S. Solomon, and J. S. Harris, Jr., Appl. Phys. Lett. 71, 1083 (1997).

${ }^{12}$ G. S. Solomon, W. Wu, J. R. Tucker, and J. S. Harris, Phys. E 2, 709 (1998).

${ }^{13}$ E. Mateeva, P. Sutter, J. C. Bean, and M. G. Lagally, Appl. Phys. Lett. 71, 3233 (1997)

${ }^{14}$ K. Kamath, N. Chervela, K. K. Linder, T. Sosnowski, H.-T. Jiang, T. Norris, J. Singh, and P. Bhattacharya, Appl. Phys. Lett. 71, 927 (1997).

${ }^{15}$ J. Tersoff, C. Teichert, and M. G. Lagally, Phys. Rev. Lett. 76, 1675 (1996)

${ }^{16}$ A. O. Kosogov, P. Werner, U. Gosele, N. N. Ledenstov, D. Bimberg, V. M. Ustinov, A. Yu, Egorov, A. E. Ahukov, P. S. Kopev, N. A. Bert, and Z. I. Alferov, Appl. Phys. Lett. 69, 3072 (1996).

${ }^{17}$ S. S. Rao, W. P. Gillin, and K. P. Homewood, Phys. Rev. B 50, 8071 (1994).

${ }^{18}$ S.-W. Ryu, I. Kim, B.-D. Choe, and W. G. Jeong, Appl. Phys. Lett. 67, 1417 (1995)

${ }^{19}$ G. P. Kothiyal and P. Bhattacharya, J. Appl. Phys. 63, 2760 (1988). 